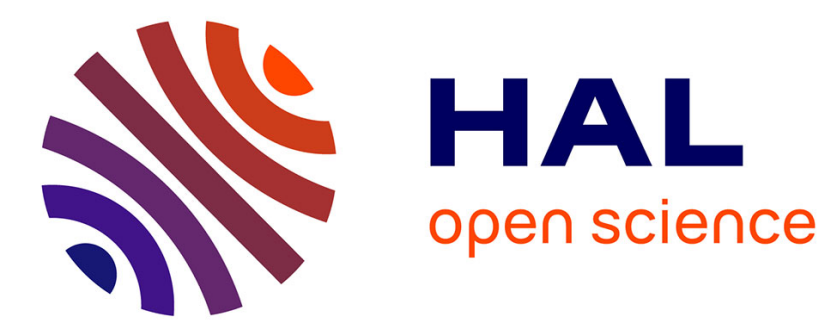

\title{
Peak-to-Average Power Ratio Reduction for OFDM Based on Dynamic Range Compression
}

\author{
Stanislaw Gorlow, Zsolt Kollár
}

\section{To cite this version:}

Stanislaw Gorlow, Zsolt Kollár. Peak-to-Average Power Ratio Reduction for OFDM Based on Dynamic Range Compression. IEEE International Symposium on Broadband Multimedia Systems and Broadcasting 2015, Jun 2015, Ghent, Belgium. hal-01165767

\section{HAL Id: hal-01165767 https://hal.science/hal-01165767}

Submitted on 20 Jun 2015

HAL is a multi-disciplinary open access archive for the deposit and dissemination of scientific research documents, whether they are published or not. The documents may come from teaching and research institutions in France or abroad, or from public or private research centers.
L'archive ouverte pluridisciplinaire HAL, est destinée au dépôt et à la diffusion de documents scientifiques de niveau recherche, publiés ou non, émanant des établissements d'enseignement et de recherche français ou étrangers, des laboratoires publics ou privés. 


\title{
Peak-to-Average Power Ratio Reduction for OFDM Based on Dynamic Range Compression
}

\author{
Stanislaw Gorlow \\ Zsolt Kollár \\ Laboratoire Bordelais de Recherche en Informatique Dept. of Broadband Infocommunication and Electromagnetic Theory \\ UMR 5800, CNRS \\ Talence, France \\ Budapest University of Technology and Economics \\ Budapest, Hungary
}

\begin{abstract}
We present a peak-to-average power ratio (PAPR) reduction method for orthogonal frequency-division multiplexing (OFDM) or similar modulation schemes based on dynamic range compression and decompression. Initially, the decompressor was developed for compressed audio signals. With regard to OFDM, the greatest benefit of the method is that it can be easily adjusted to the system requirements and a tradeoff can be found between the PAPR gain and signal distortion. Practically, it requires no additional side information at the receiver. In a pilot experiment, we evaluate the method using four different metrics and give a brief interpretation of the obtained results.
\end{abstract}

\section{INTRODUCTION}

As the wireless and mobile communication continues to evolve and new standards are being introduced, limits are pushed towards even more efficient data transmission rates and a higher spectral efficiency. ${ }^{1}$ This also relates to higher throughput, more efficient use of power amplifiers and better power consumption. In the most advanced high-speed wireless communication systems, such as the second-generation Digital Video Broadcasting (DVB) or Long-Term Evolution (LTE), the main modulation format is orthogonal frequency-division multiplexing (OFDM) [1]. OFDM is a spectrally efficient multicarrier modulation scheme. Aside from many benefits, one of its biggest disadvantages is the signal's relatively high peak-to-average power ratio (PAPR), which requires power amplifiers with a wide linear range, operating less efficiently. Otherwise, nonlinear distortion may appear, which would decrease the system performance and might as well introduce out-of-band radiation.

Various PAPR reduction techniques were developed and further improved over the past years. Some of them require additional side information, some lead to a decrease of the data rate, others consume more power [2], [3]. In this paper, we present a novel PAPR reduction approach for OFDM signals based on dynamic range compression (DRC) and decompression (DRD). Essentially, this technique does not require any side information, which means that the data rate can be maintained. Solely a certain amount of distortion is introduced in exchange for a relatively high PAPR gain. DRC is used in music production and radio broadcasting, but it can also be applied to OFDM signals, as we will demonstrate.

\footnotetext{
${ }^{1}$ See also the Future of Broadcast Television initiative (http://fobtv.org)
}

The paper is organized as follows. In Section II, we briefly discuss the OFDM modulation scheme and the related PAPR problem. In Sections III and IV, we describe the compressor and the decompressor that we use for PAPR reduction. In the following Section V, we evaluate the proposed technique by means of the achieved PAPR gain, the resulting modification of the signal's power spectrum, the modulation error ratio and the magnitude of the error vector. Section VI concludes the paper.

\section{OFDM AND PAPR}

\section{A. Orthogonal Frequency-Division Multiplexing}

Orthogonal frequency-division multiplexing (OFDM) is a popular multicarrier modulation scheme. The digital baseband signal with $N$ subcarriers for a single symbol is generated using the inverse (fast) Fourier transform according to

$$
x(n)=\sum_{k=0}^{N-1} X(k) \mathrm{e}^{\mathrm{j} \frac{2 \pi}{N} n k} \quad n=0,1, \ldots, N-1,
$$

where $X(k) \in \mathbb{C}$ is the modulation value of the $k$ th subcarrier taken from a given alphabet and $\mathrm{j}$ is the imaginary unit. As a rule, an OFDM sequence is extended by a cyclic prefix to combat multipath propagation.

One may say that the main disadvantage of OFDM lies in its sensitivity to nonlinear distortion. As an OFDM signal is the sum of a large number of independently modulated subcarriers, the resulting signal has a nearly normal distribution. For this, very high peaks appear. To avoid nonlinear distortion over the entire signal range, the power amplifier at the transmitter must operate at a lower level, and so less efficiently. This prevents the peaks from reaching into the nonlinear region of an analog amplifier. If the power amplifier is overdriven beyond its linear region, nonlinear distortion will appear in the modulated signal, which may decrease the overall performance.

\section{B. Peak-to-Average Power Ratio}

One way of measuring the fluctuation of an OFDM signal is by the peak-to-average power ratio (PAPR) defined as

$$
\operatorname{PAPR}(\mathrm{dB})=10 \log _{10} \frac{\max P_{\text {sig }}}{P_{\text {sig }}(\mathrm{RMS})},
$$

where the average signal power is measured by the root mean square (RMS). During evaluation, in place of the PAPR itself 
we will consider its complementary cumulative distribution function $(\mathrm{CCDF})$.

\section{DYNAMIC RANGE COMPRESSION}

Dynamic range compression (DRC) is a sound processing technique that attenuates loud sounds and/or amplifies quiet sounds, which in consequence leads to a reduction of an audio signal's dynamic range [4]. The latter is sometimes defined as the difference between the loudest and the quietest sound measured in decibel $(\mathrm{dB})$. A more appropriate way to express the dynamic range is using the crest factor, or the PAPR when expressed in $\mathrm{dB}$. Also note that throughout the paper we mean downward compression when we speak in vague terms of "compression". Downward compression attenuates sounds above a given threshold while leaving sounds below the threshold unchanged. Fig. 1(a) shows such a digital compressor model. It was adapted to OFDM based on an error analysis of a more generic model given the properties of OFDM signals. Moreover, as we now consider complex baseband signals, the in-phase and quadrature (IQ) components are processed independently. This corresponds to the case where a stereo audio signal is processed without stereo linking.

The compressor's operation is as follows. The input signal is split and a copy is sent to the side chain. The detector calculates the level of the sidechain signal using the root mean square (RMS) as a measure, while its reactivity to the current input is controlled by a time constant. This sidechain signal level is compared to the threshold level and, for the case it exceeds the threshold, a gain factor is calculated which corresponds to the ratio of the input level to the output level. At the end of the side chain, the input signal is multiplied by the gain. The difference between the original signal's PAPR value and the compressed signal's PAPR value represents the PAPR gain. The building blocks of the proposed OFDM compressor are detailed below.

\section{A. RMS Detector}

The RMS detector builds upon a first-order lowpass filter. And so, the sound level or envelope $v(n)$ of the input signal $x(n)$ is computed according to

$$
\begin{aligned}
& \tilde{x}(n)=\alpha x^{2}(n)+(1-\alpha) \tilde{x}(n-1) \\
& v(n)=\sqrt{\tilde{x}(n)} .
\end{aligned}
$$

The smoothing factor $\alpha, 0<\alpha \leqslant 1$, determines the reactivity of the detector. The relation between the smoothing factor $\alpha$ and a time constant $\tau$ is given by

$$
\alpha=1-\exp \left(-2.2 \frac{T_{s}}{\tau}\right),
$$

where $T_{s}$ is the sampling period and $\exp (\cdot)$ is the exponential function.

\section{B. Gain Computer}

The static nonlinearity in the gain computer is a continuous piecewise linear function in the logarithmic domain:

$$
F(n)= \begin{cases}-S[V(n)-L] & \text { if } V(n)>L, \\ 0 & \text { otherwise, }\end{cases}
$$

where $L$ is the threshold in $\mathrm{dB}$,

$$
V(n)=20 \log _{10} v(n),
$$

and $S$ is the slope. $S$ is derived from the compression ratio $R$ according to

$$
S=1-\frac{1}{R}
$$

In the linear domain, (5) corresponds to

$$
f(n)= \begin{cases}\kappa v^{-S}(n) & \text { if } v(n)>l \\ 1 & \text { otherwise }\end{cases}
$$

with

$$
\begin{aligned}
l & =10^{L / 20}, \\
\kappa & =l^{S} .
\end{aligned}
$$

The linear scale factor $f(n)$, or gain, is finally applied to the input signal $x(n)$ to obtain the compressed output signal:

$$
y(n)=f(n) x(n) .
$$

\section{DYNAMIC RANGE DECOMPRESSION}

Dynamic range decompression (DRD) is a newly developed technique that allows to reverse dynamic range compression [5]. Knowing the compressor model and its parameters (reactivity, threshold, and ratio), the corresponding decompressor completely and accurately inverts dynamic range compression giving back dynamics to the compressed signal. The decompressor has as input the compressed signal and the parameters of the compressor model. It uses these to generate a signal which, if it were compressed with the given parameters, would closely correspond to the original signal before compression. Therefore, it can be used to undo compression with minimal metadata or no metadata at all, if the parameters are fixed [6]. It requires a relatively low computational effort and has zero delay due to pure time-domain processing. We intend to apply the decompressor to a compressed OFDM signal before demodulation at the receiver, see Fig. 1(b). For the internals see below.

\section{A. RMS Predictor}

To predict when compression was active, formally $v(n)>l$ in (8), we require an instantaneous estimate for the envelope value $v(n)$. In the opposite case, where $v(n) \leqslant l$, it can be noted that $f(n)=1$, and so

$$
x(n)=y(n) .
$$

The sound level of the input signal at instant $n$ is therefore

$$
v(n)=\sqrt{\alpha y^{2}(n)+(1-\alpha) \tilde{x}(n-1)},
$$

which must be greater than the threshold $l$ for decompression to set in. 
(a) OFDM IQ compressor

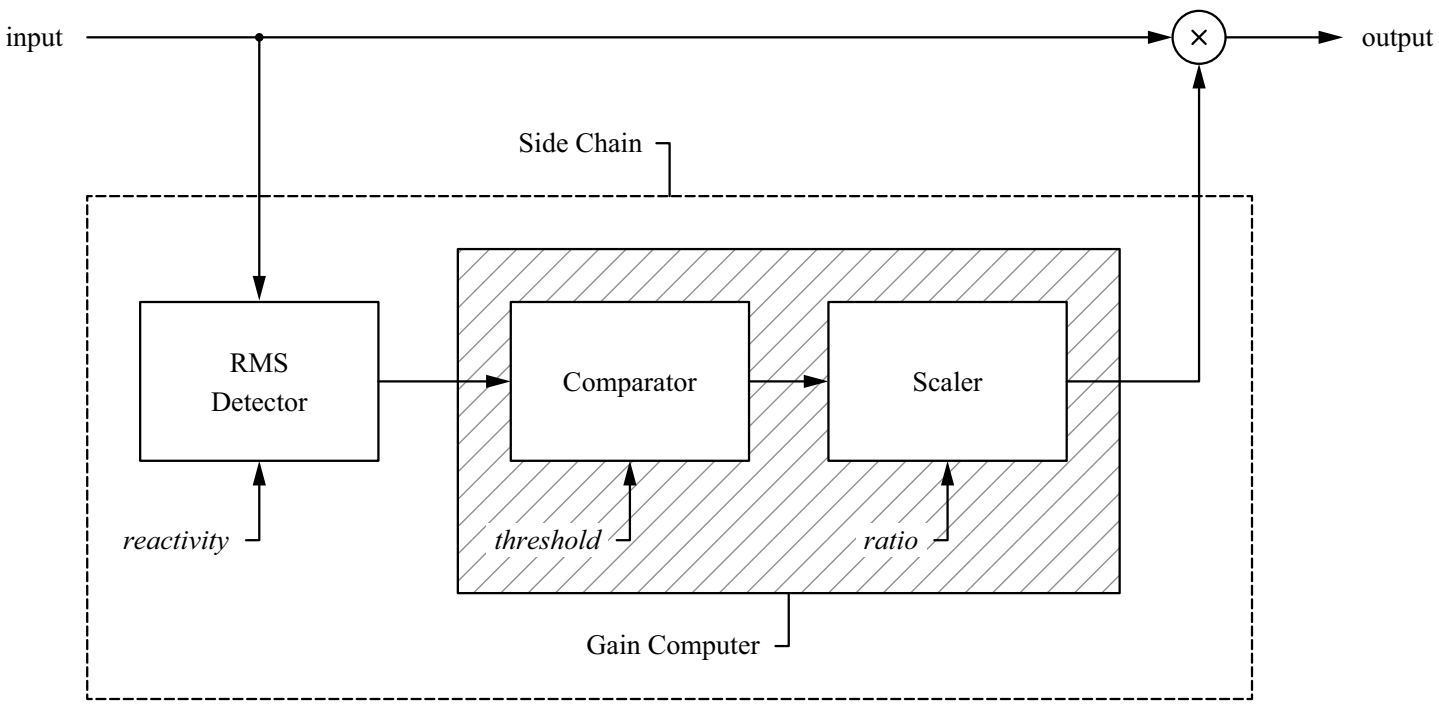

(b) OFDM IQ decompressor

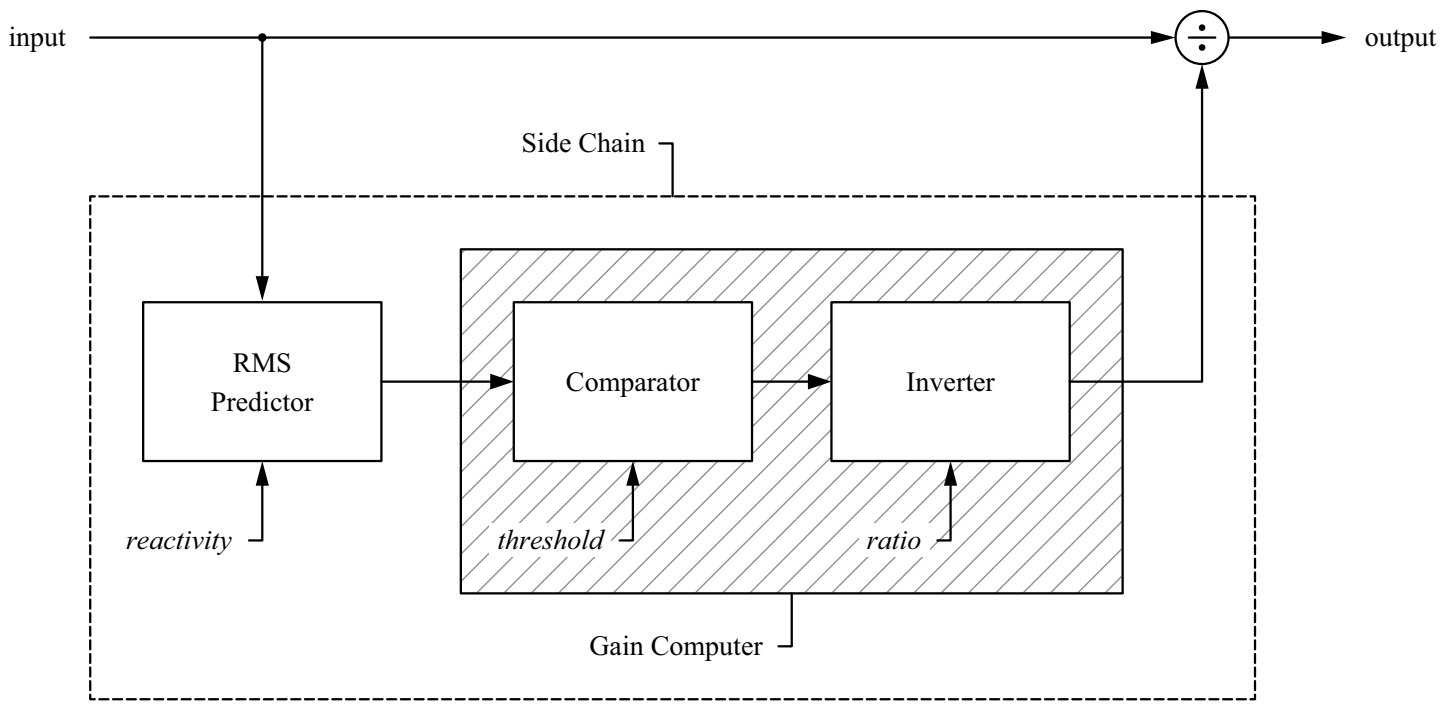

Fig. 1. Logical structure of (a) an OFDM IQ compressor and (b) an OFDM IQ decompressor.

\section{B. Inverter}

The decompressor in Fig. 1(b) can be characterized by the nonlinear function

$$
\begin{gathered}
\zeta(v)=\left[\alpha \kappa v^{-S}(n)\right]^{2}\left[v^{2}(n)-(1-\alpha) \tilde{x}(n-1)\right] \\
-\alpha y^{2}(n) .
\end{gathered}
$$

The root $v_{0}$ of $\zeta(v)$ represents the sought-after envelope value $v(n)$ during compression. Once $v_{0}(n)$ is found ${ }^{2}$, the current values of $\tilde{x}$ and $|x|$ are updated according to

$$
\begin{aligned}
\tilde{x}(n) & =v_{0}^{2}(n), \\
|x(n)| & =\sqrt{\frac{\tilde{x}(n)-(1-\alpha) \tilde{x}(n-1)}{\alpha}},
\end{aligned}
$$

\footnotetext{
${ }^{2} \mathrm{~A}$ fast root-finding algorithm for (14) is given in [5].
}

if $v(n)>l$, or else

$$
\begin{aligned}
|x(n)| & =|y(n)|, \\
\tilde{x}(n) & =\alpha|x(n)|^{2}+(1-\alpha) \tilde{x}(n-1) .
\end{aligned}
$$

The decompressed sample is then computed as

$$
z(n)=\operatorname{sgn}(y)|x(n)|,
$$

where $\operatorname{sgn}(\cdot)$ is the sign function.

\section{PROOF OF CONCEPT}

Due to the fact that an OFDM signal exhibits very noiselike characteristics, the time constant in our model must be set to a very small value for the compressor to be able to react fast enough. Small values, however, lead to an increased decompression error, as explained in [5]. In what follows, the time constant is denoted by $\tau$. With regard to the threshold, it 
can be set arbitrarily but below the peak magnitude of the input signal. We set it to the mean input power level (RMS). The compression ratio $R$ determines the PAPR gain. The following rule applies: the higher the compression ratio, the higher the PAPR gain. It should be kept in mind, however, that a higher compression ratio makes the inversion less accurate, see [5]. This means that there is a tradeoff between the PAPR gain and the accuracy of the decompressed signal. To shine a light on that tradeoff, we conduct a pilot experiment.

\section{A. Protocol}

The operating frequency of the OFDM signal is $48 \mathrm{kHz}$, which is typical for audio, but could be set to any other frequency as well. The guard interval is equal to $25 \%$, which means that the signal's effective bandwidth is $18 \mathrm{kHz}$. The original OFDM signal is upsampled by a factor of 4 to $192 \mathrm{kHz}$ and DRC is applied to both of the signals (critically sampled and oversampled) for comparison. The two compressed signals are evaluated in terms of their complementary cumulative distribution functions (CCDFs) of the PAPR and their power spectral densities (PSDs). Then, the two compressed signals are decompressed, downsampled if necessary, and demodulated. In a final step, the modulation error ratios (MERs) and the induced error vector magnitudes (EVMs) are computed based on the ideal and demodulated quadrature phase-shift keying (QPSK) symbol sequences.

\section{B. Performance Measures}

To be able to assess the performance of the proposed scheme and the introduced distortion, four measures are considered. The most important one for us is the PAPR gain. It is defined as the ratio of the CCDFs of the modulated and the compressed signal at a probability of $10^{-2}$, i.e.

$$
\operatorname{PAPRG}(\mathrm{dB})=10 \log _{10} \frac{\operatorname{PAPR}_{0.01}(\mathrm{OFDM})}{\operatorname{PAPR}_{0.01}(\mathrm{DRC})}
$$

To assess the signal distortion, we measure the increase in out-of-band radiation (OBR). We define it as the ratio of the out-of-band-to-in-band power ratios of the compressed and the modulated signal. Formally,

$$
\mathrm{OBR}(\mathrm{dB})=10 \log _{10} \frac{P_{\text {out }}(\mathrm{DRC})}{P_{\text {in }}(\mathrm{DRC})} / \frac{P_{\text {out }}(\text { OFDM })}{P_{\text {in }}(\text { OFDM })} .
$$

Furthermore, for data communication systems, the MER and the EVM are very common measures. They indicate how much the demodulated signal is distorted in comparison to the ideal signal. These metrics are defined as ratios of the signal power and the error power and are reciprocal. Whereas both use the RMS power of the error signal, the EVM uses the maximum power in the ideal signal constellation, i.e.

$$
\operatorname{MER}(\mathrm{dB})=10 \log _{10} \frac{P_{\text {sig }}(\mathrm{RMS})}{P_{\text {err }}(\mathrm{RMS})}
$$

and

$$
\operatorname{EVM}(\mathrm{dB})=10 \log _{10} \frac{P_{\text {err }}(\mathrm{RMS})}{\max P_{\text {sig }}},
$$

respectively.

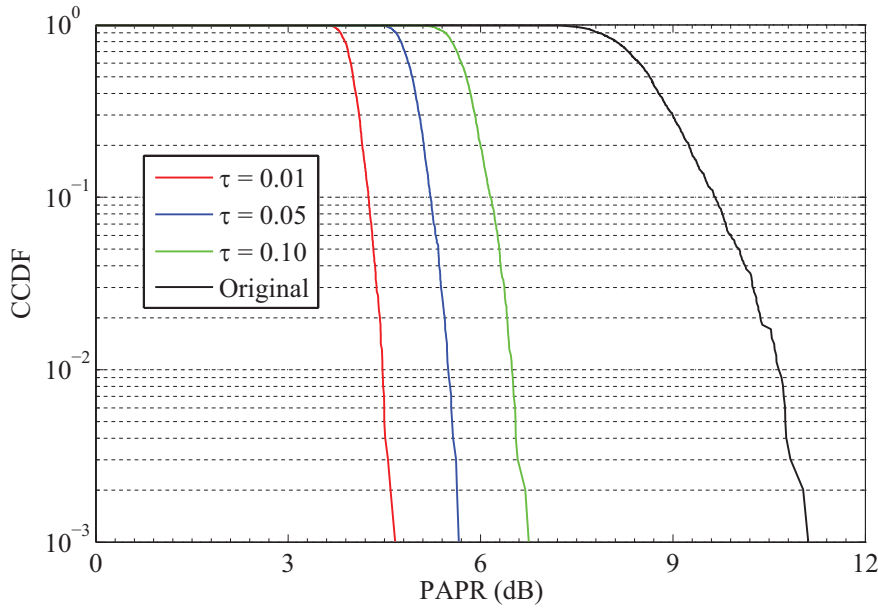

Fig. 2. CCDF of the PAPR of the transmitted OFDM signal for different $\tau$-values $(R=5: 1)$. The operating frequency is $48 \mathrm{kHz}$.

\section{Experimental Results}

Our primary objective was to move the CCDF of the PAPR further to the left, i.e. to reduce the probability of high peaks in the signal by means of DRC. This effect can indeed be observed in Fig. 2. More precisely, it can be seen that the PAPR gain is improving for smaller values of $\tau$. For a value of 0.10 the PAPR gain is around $4.2 \mathrm{~dB}$. For values below 0.05 our approach is close to the state of the art, as the PAPR gain increases from 5.2 to $6.2 \mathrm{~dB}$ for $\tau=0.01$. Moreover, it should be noted that the state of the art, which is capable of surpassing the $3.0 \mathrm{~dB}$ margin, in general has a much higher computational complexity [7], [8]. The simplified, low-complexity PAPR reduction techniques have a much lower gain. Fig. 5(a) confirms that the PAPR gain grows logarithmically with the compression ratio. Compared to the $48-\mathrm{kHz}$ signal, the upsampled $192-\mathrm{kHz}$ signal loses some performance. The PAPR loss is less dramatic for the smallest $\tau$-value, see Fig. 5(b).

To visualize the out-of-band radiation that occurs due to the application of DRC, we plot the PSDs of the compressed OFDM signals versus the original in Fig. 3. It can be seen that some of the in-band signal power is spilled to the sides. The effect grows when $\tau$ takes on smaller values. The exact impact of out-of-band radiation, however, depends on the application and the size of the guard interval. ${ }^{3}$ And so, it cannot be foretold without context. In any case, Fig. 5(c) shows that OBR grows with the compression ratio and also increases reciprocally to $\tau$. One interesting fact to notice in Fig. 5(d) is that upsampling reduces the OBR for the smallest $\tau$-value irrespective of the compression ratio, but noticeably augments the OBR for the largest value of $\tau$.

At last, the signal distortion after demodulation and prior decompression is illustrated in the form of the constellation diagram. Fig. 4 confirms what could be expected: a greater

\footnotetext{
${ }^{3}$ The acceptable amount of out-of-band radiation is usually determined by the communication system's spectral mask.
} 


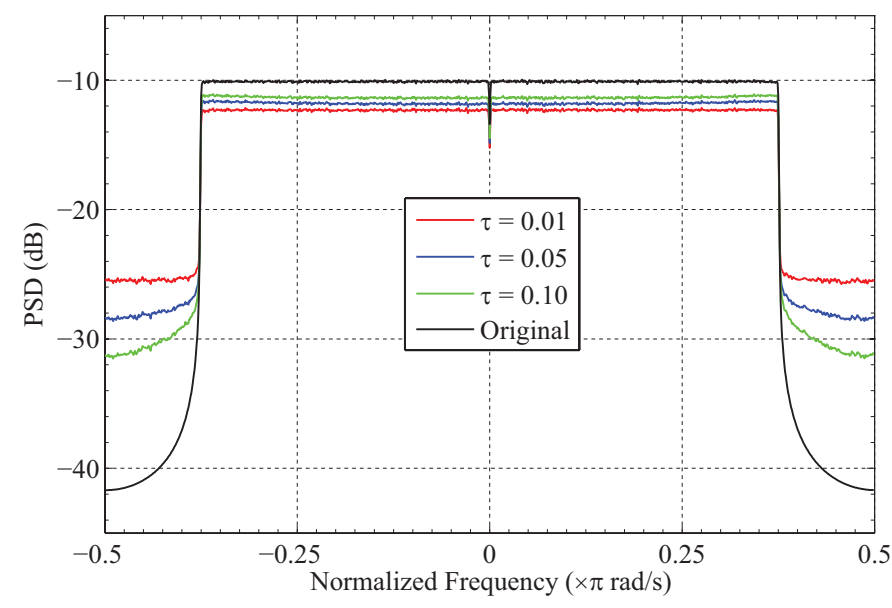

Fig. 3. PSD of the transmitted OFDM signal for different $\tau$-values $(R=5$ : 1). The operating frequency is $48 \mathrm{kHz}$.

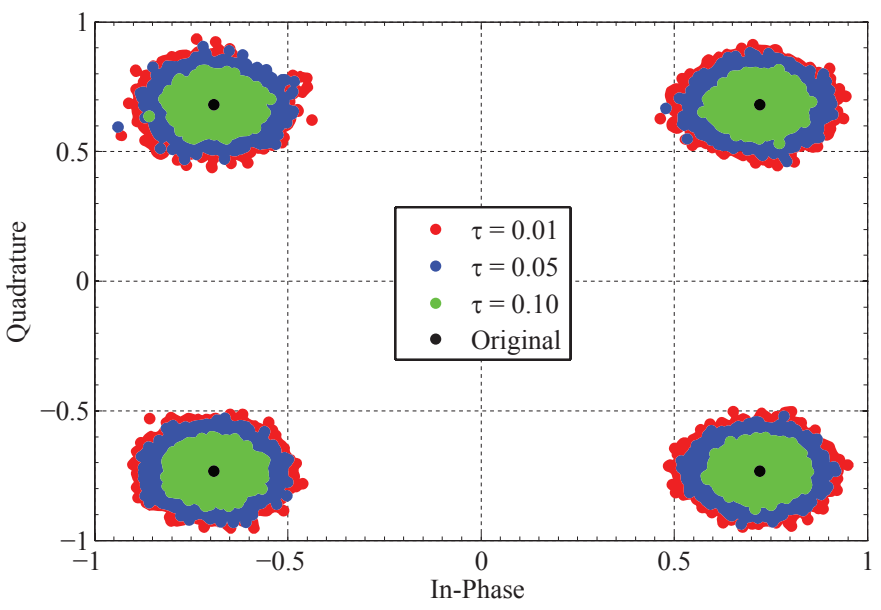

Fig. 4. Constellation diagram of the demodulated OFDM signal for different $\tau$-values $(R=5: 1)$. The operating frequency is $48 \mathrm{kHz}$.

PAPR gain comes at the cost of a larger inversion error, which again may lead to a higher bit error rate (BER). In our experiment, where the channel is absent, the BER is zero. It is because the symbol clouds do not overlap and are hence perfectly distinguishable from each other. Looking at Fig. 5(e) one can see that even for the parameters that bear the highest gain, the MER is still as high as $23 \mathrm{~dB}$. The MER drops with the gain and with the compressor's reactivity. The same is true for the EVM in Fig. 5(g), respectively. Finally, Figs. 5(f) and 5(h) convey that upsampling is somewhat counterproductive for a very short $\tau$, but has a very positive effect otherwise.

\section{CONCLUSION}

The paper shows that dynamic range compression and decompression can be used to successfully reduce the relatively high peak-to-average power ratio of an OFDM signal. Since nothing comes for free, the proposed scheme is subject to a tradeoff between the gain and signal distortion in the form of out-of-band radiation and inversion errors. Our performance analysis suggests that the best result is achieved for a compression ratio between $3: 1$ and $4: 1$, a very short time constant, and when the OFDM signal is upsampled to a higher rate. In that case, the achievable PAPR gain lies between 5 and $6 \mathrm{~dB}$, while the modulation error ratio is above $24 \mathrm{~dB}$. To conclude, it looks like the proposed scheme can challenge the state of the art. A comparison with other existing techniques would be of great benefit.

\section{ACKNOWLEDGMENT}

The authors would like to thank Aquitaine Science Transfert for letting them use the Dynastore-X software during tests.

\section{REFERENCES}

[1] R. van Nee and R. Prasad, OFDM for Wireless Multimedia Communications, 1st ed. Norwood, MA, USA: Artech House, 2000.

[2] S. H. Han and J. H. Lee, "An overview of peak-to-average power ratio reduction techniques for multicarrier transmission," Wireless Commun., vol. 12, no. 2, pp. 56-65, Apr. 2005.

[3] T. Jiang and Y. Wu, "An overview: Peak-to-average power ratio reduction techniques for OFDM signals," IEEE Trans. Broadcast., vol. 54, no. 2, pp. 257-268, Jun. 2008.

[4] U. Zölzer, DAFX: Digital Audio Effects, 2nd ed. John Wiley \& Sons, 2011.

[5] S. Gorlow and J. D. Reiss, "Model-based inversion of dynamic range compression," IEEE Audio, Speech, Language Process., vol. 21, no. 7, pp. 1434-1444, Jul. 2013.

[6] S. Gorlow, J. D. Reiss, and E. Duru, "Restoring the dynamics of clipped audio material by inversion of dynamic range compression," in IEEE $B M S B$, Jun. 2014, pp. 1-5.

[7] M. Deumal, A. Behravan, and J. L. Pijoan, "On cubic metric reduction in OFDM systems by tone reservation," IEEE Trans. Commun., vol. 59 , no. 6, pp. 1612-1620, Apr. 2011.

[8] B. Horváth, Z. Kollár, and P. Horváth, "Bridging the gap between optimal and suboptimal ACE PAPR reduction scheme for OFDM," in RADIOELEKTRONIKA, Apr. 2014, pp. 1-4. 
(a) Peak-to-average power ratio gain

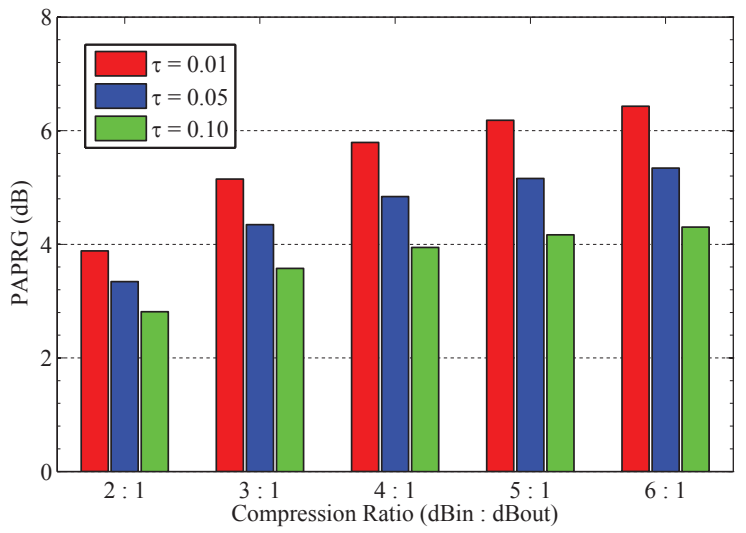

(c) Out-of-band radiation

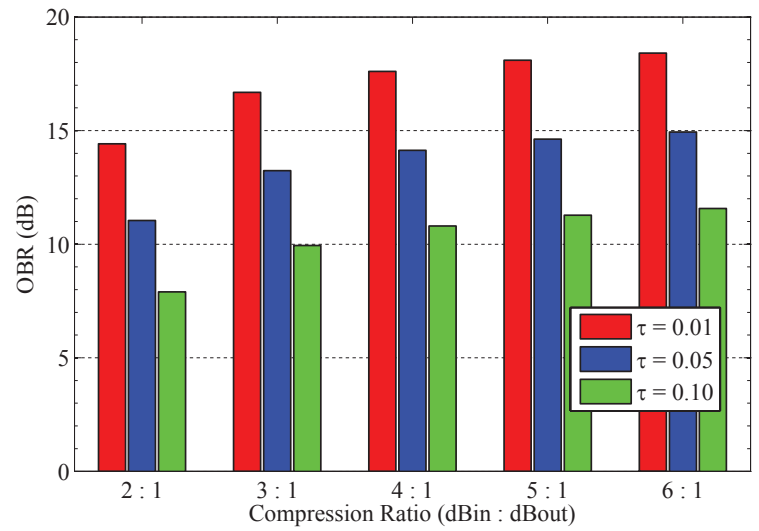

(e) Modulation error ratio

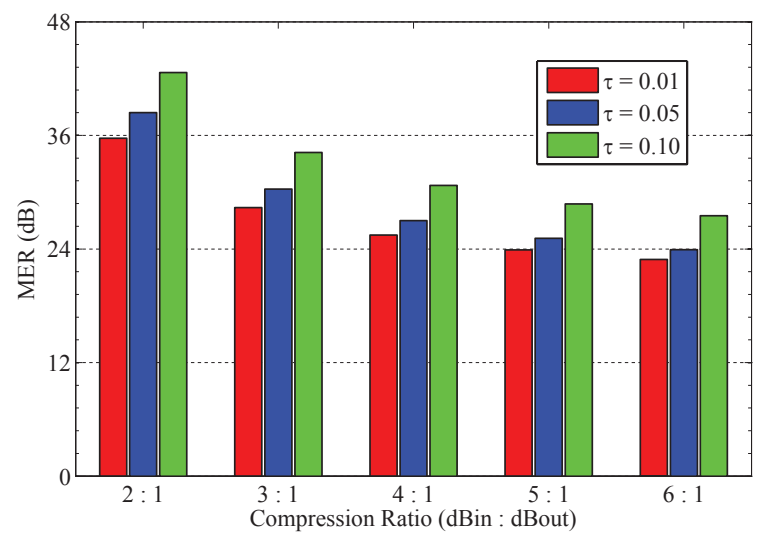

(g) Error vector magnitude

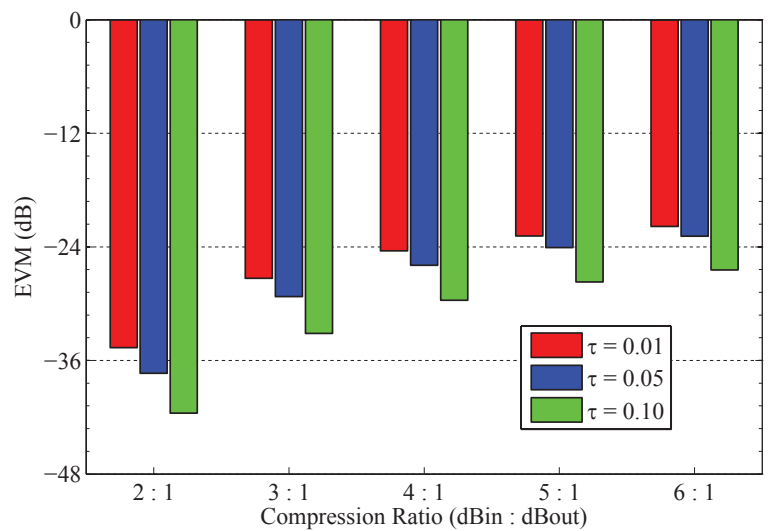

(b) Variation in peak-to-average power ratio gain

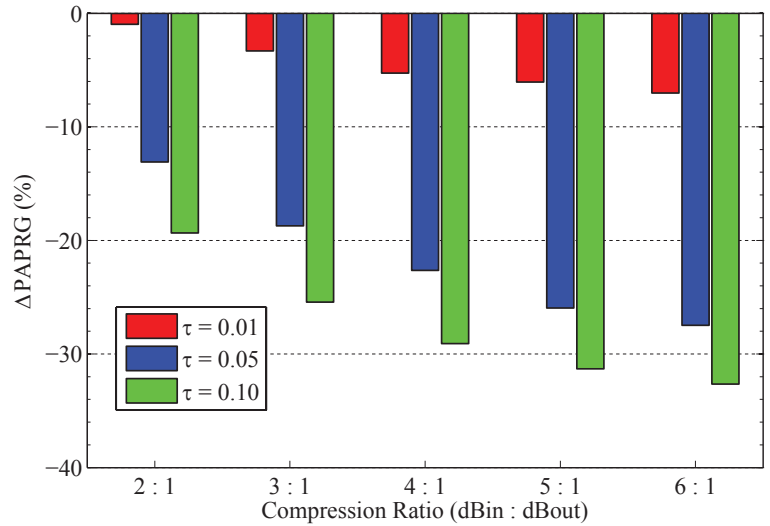

(d) Variation in out-of-band radiation

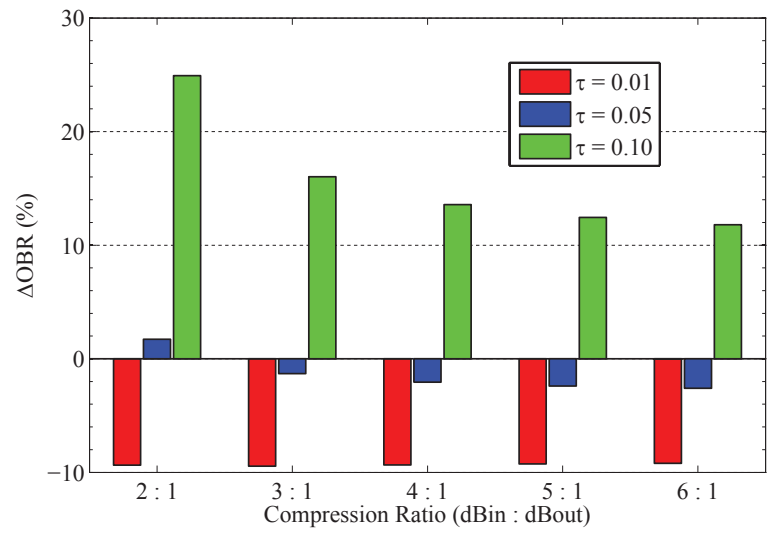

(f) Variation in modulation error ratio

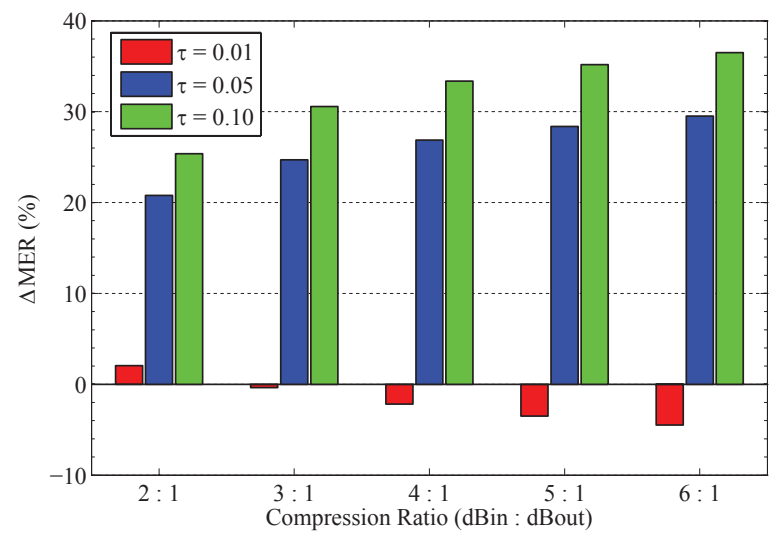

(h) Variation in error vector magnitude

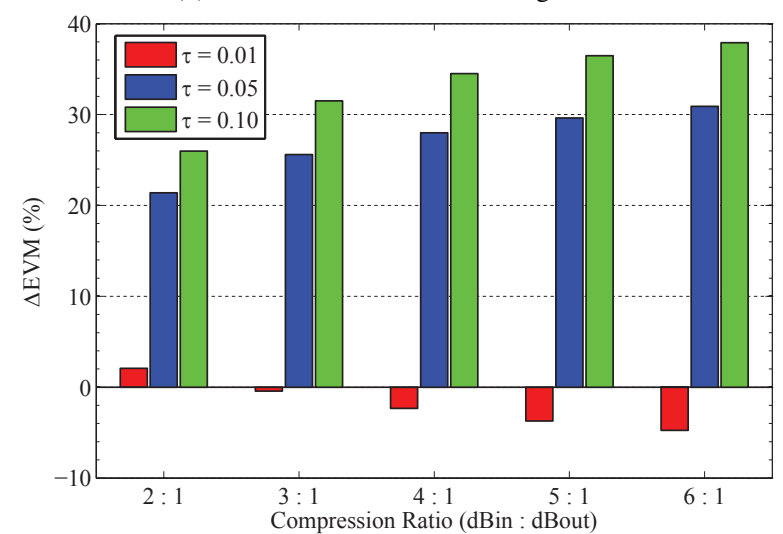

Fig. 5. Performance of the compressor/decompressor at $48 \mathrm{kHz}$ (left column) and the performance variation due to upsampling to $192 \mathrm{kHz}$ (right column) for various compression ratios and different time constants. 